\title{
Vortexing Flow Patterns in a Water Model of Slab Continuous Casting Mold
}

\author{
Baokuan LI and Fumitaka TSUKIHASHI' \\ Department of Thermal Engineering, School of Materials and Metallurgy, Northeastern University, Shenyang, 110004 China. \\ 1) Department of Advanced Materials, Graduate School of Frontier Science, The University of Tokyo, 5-1-5 Kashiwanoha, \\ Kashiwa, Chiba 277-8561 Japan.
}

(Received on June 18, 2004; accepted in final form on October 8, 2004)

\begin{abstract}
A water model experiment was conducted to observe the vortexing flow in the steel slab continuous casting mold, the snake- shaped Plexiglas mold was designed to simulate the actual caster. The camera was used to record the flow patterns, which were visualized by injecting the black sesames into water. The changes of shape of single vortex and two vortices with time have been observed during experiments. A numerical model has been developed to analyze the vortexing flow, which may be produced by moving the submerged entry nozzle from center to off-center in the slab continuous casting of steel. According to the numerical results, the vortexing flow is resulted from three-dimensional biased flow in the mold. A vortex is located at the low velocity side adjacent to the submerged entry nozzle. The vortex strength depends on the local horizontal velocity of fluid and decreases gradually with distance from the free surface. The vortexing zone size depends on the biased distance of the submerged entry nozzle, and intensity of the vortexing flow depends on the casting speed of the continuous caster.
\end{abstract}

KEY WORDS: continuous casting; water model; simulation; vortexing flow.

\section{Introduction}

With increasing the production of continuous caster with higher casting speeds, it becomes important to keep a stable and relatively quiescent meniscus in the slab continuous casting mold. However, molten steel flow in a mold is unstable, and different types of flow patterns exist, especially for high casting speed. These include off-center placing of a submerged entry nozzle (SEN), periodic oscillations and random fluctuations due to argon gas injection and turbulence, etc. Vortexing flow is generally associated with draining of a metallurgical vessel. ${ }^{1)}$ A slag-entraining vortex appears as the bath reaches a critical depth during drainage. This vortex is composed of a downward flow toward the outlet and a planar vortex (two-dimensional rotation of fluid). Vortices are able to cause the mold powder entrapment; the entrapped flux may be carried deeply down into the mold by downward flows and results in uncleanness of steel.

Water modeling studies have revealed existence of the vortex in the slab mold adjacent to the SEN. ${ }^{1-3)}$ Wang $^{1)}$ reported the appearance of a vortex adjacent to the SEN. The results from surface quality and sliver defects analyses revealed that most defects occurred at the middle of the slab width, which suggested that the vortex may be a source of slag entrapment and sliver defects. $\mathrm{He}^{2)}$ concluded that vortex existence in the mold was the result of biased flow between the SEN ports generated by the effects of slide gate, nozzle clogging, and turbulent flow, etc. Using a series of flow visualization experiment, Gebhard et al. ${ }^{3)}$ found that this vortexing phenomena are casused by the same mecha- nism as that commonly termed a 'bathtub vortex', and the surface velocity affected the depth and stability of the vortex. The study of Honeyands and Herbertson ${ }^{4)}$ shows the biased flow at high casting speed caused by transients. Gupta and Lahri $\left.{ }^{5}\right)$ illustrate the biased flow caused by geometrical conditions of the mold. Huang and Thomas ${ }^{6)}$ studied the transition from biased flow to symmetrical steady flow by changing nozzle inlet conditions, but they did not mention vortexing phenomenon. Yoshida et al. ${ }^{7)}$ proposed the mechanisms of mold powder entrapment, i.e. it is related to the pressure difference around the nozzle.

Although some researcher ${ }^{1-3)}$ have studied the vortexing flow in the continuous casting mold using the water model, the true picture of vortexing flow is still not reported and the mathematical model which can describe this phenomenon is not reported yet.

This first objective of the present work is to observe and record the live vortexing flows in the slab continuous casting process of steel using the water model. The second objective is to develop a mathematical model, which simulates the vortexing flow in the mold, and then analyze the vortexing flow characteristics and the effects of the some geometrical and casting conditions on vortexing flow in a slab continuous casting mold.

\section{Experiment}

A physical model of the thin slab caster mold with fullscale was constructed using Plexiglas. Water was used as modeling fluid, with satisfying both the Reynolds and Froude number similarity criteria at any given volumetric 


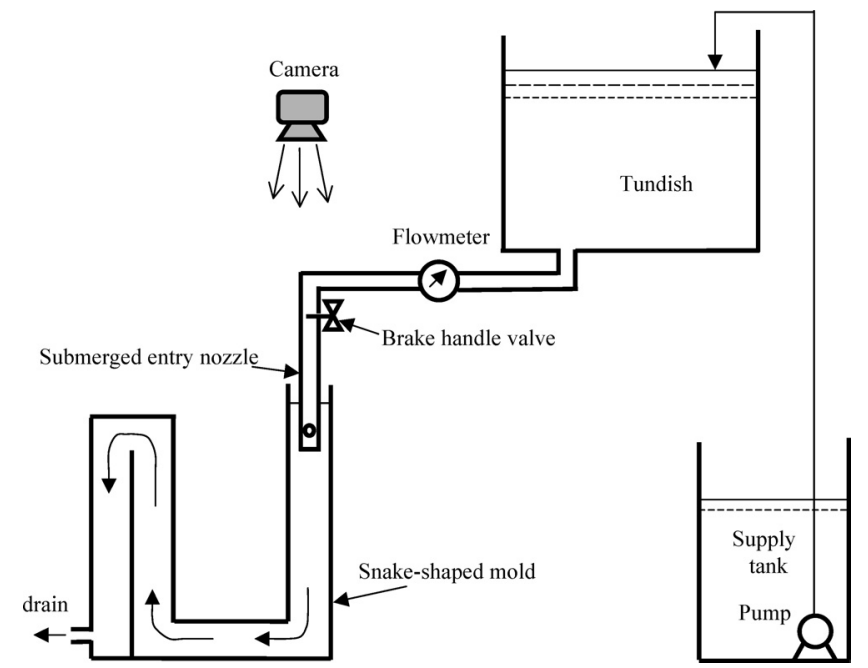

Fig. 1. Schematic diagram of the water model flow system.

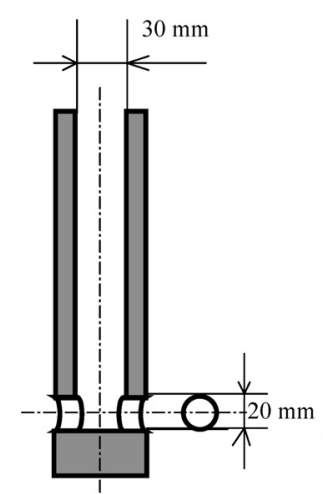

(d)

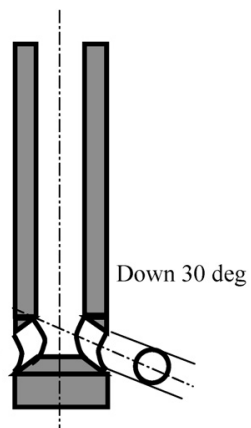

(b)

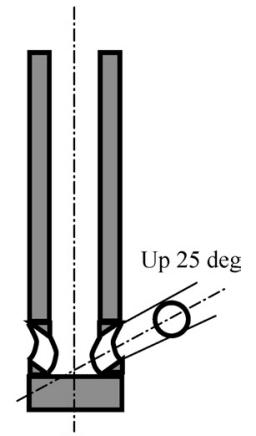

(c)
Fig. 2. The nozzle configurations.

Table 1. Geometrical parameters and physical properties of fluid in simulation.

\begin{tabular}{ll}
\hline Parameters & Values \\
\hline Mold size, $\mathrm{mm}$ & 500 (width) $\times 70$ (thickness) $\times 1000$ (length) \\
Nozzle size, $N \times W, \mathrm{~mm}$ & 24 (thickness) $\times 30$ (width) \\
Nozzle outlet size, $\mathrm{mm}$ & 18 (width) $\times 20$ (height), (Two outlets) \\
Depth of nozzle, $L_{\mathrm{n},} \mathrm{mm}$ & 120 \\
Casting speed, $\mathrm{m} / \mathrm{min}$ & $3.0,4.0$ \\
Outflow angle, ${ }^{0}$ & 0 \\
Density of liquid, $\mathrm{kg} / \mathrm{m}^{3}$ & 1000 \\
Viscosity of liquid, $\mathrm{N} \cdot \mathrm{s} / \mathrm{m}^{2}$ & $1.0 \times 10^{-3}$ \\
Biased distance $e_{1}, \mathrm{~mm}$ & $5,15(1 \%$ and $3 \%$ of slab width) \\
Biased distance $e_{2}, \mathrm{~mm}$ & $5,7.5(7 \%$ and $11 \%$ of slab thickness) \\
\hline
\end{tabular}

throughout. The water model system is shown in Fig. 1, Fig. 2 exhibits three kinds of nozzle configuration. The mold parameters are shown in Table 1. In order to observe easily the flow field in the upper strand, a snake-shaped model was designed to allow the simulation of flow in the mold and the upper strand, the snake-shaped mold may make the flow field in mold be analogous to practical operation in the case of occupying the smaller space. A brake handle valve was used to control the flow rate from $1.728 \times 10^{-3}$ to $2.16 \times 10^{-3} \mathrm{~m}^{3} / \mathrm{s}$, corresponding casting speed from 3 to $4 \mathrm{~m} / \mathrm{min}$. The flow patterns in the free sur-

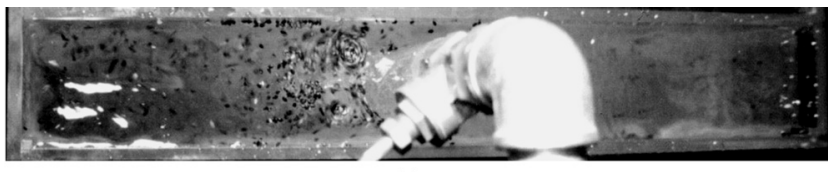

(a)

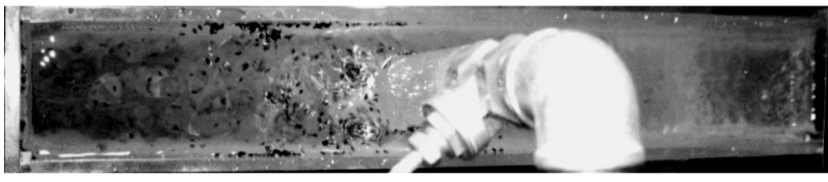

(b)

Fig. 3. Observed two vortices in free surface, $V_{\mathrm{C}}=3 \mathrm{~m} / \mathrm{min}$.

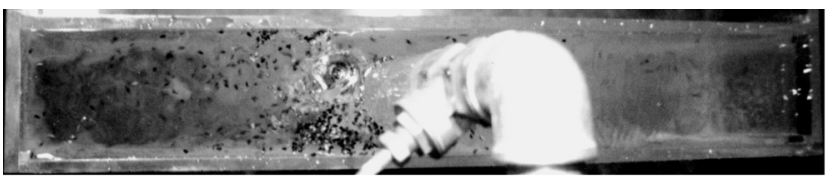

(a)

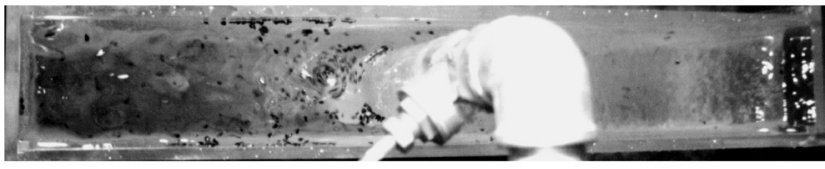

(b)

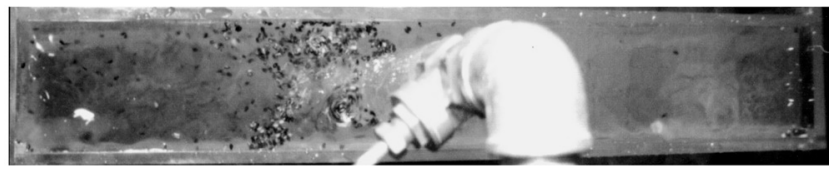

(c)

Fig. 4. Observed a vortex in free surface and located at (a) side toward the front of the SEN, (b) paralleling the SEN and (c) back of the SEN, $V_{\mathrm{C}}=3 \mathrm{~m} / \mathrm{min}$.

face were recorded by the camera and video tape recorder from the upper side.

The flow visualizations were conducted by injecting black sesames at the fluid surface of the mold. Figure 3 shows the photographs of the two vortices formation that occur once in a while and durative time is short. In the most cases, only one vortex takes place and position varies with the transient and operating conditions as shown in Figs. 4(a) through 4(c), since the flow is also biased in thickness direction. Although the geometrical and operating conditions were made strictly symmetrical, the vortices cannot be avoided yet, i.e. the vortex formation occurred near the SEN under the normal casting condition. When the SEN is located at the center of the mold, either single vortex or two vortices formed close to the SEN. These vortices are unsteady and tend to travel from a side to another of the SEN, and all vortices rotated inward. The position of vortices around the SEN depends on flow conditions in the mold. When the SEN is off-centrally located, a strong biased flow and the vortices are taken place and the vortices still move around the SEN. With the outflow angle of the SEN change from downward to upward, the trend toward the vortices formation becomes stronger. The lengths of vortex are exhibited in the vertical view using the black sesames in Fig. 5. The intensity of vortexing flow increases with the increasing of off-centerness of nozzle and flow rate of fluid. Moreover, three nozzle configurations are used to observe the vortexing flows in experiments and the vortexing flows 

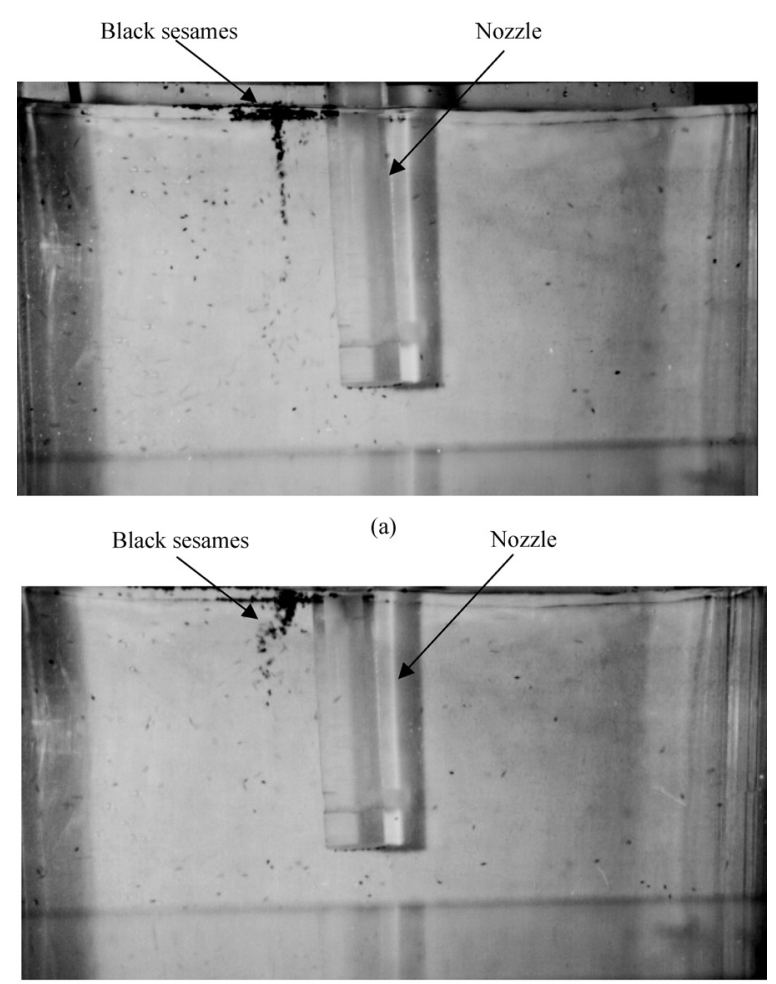

(b)

Fig. 5. The vortex displayed by the black sesames near the SEN, $V_{\mathrm{C}}=3 \mathrm{~m} / \mathrm{min}$. (a) and (b) are vortex line in vertical view.

can be found in all cases. The trend toward the vortices formation becomes strong with the outflow angle of the SEN change from downward to upward.

\section{Mathematical Model}

\subsection{Formulation Description}

A finite difference model, which focuses on the slab continuous casting of steel with a $1 \mathrm{~m}$ length of liquid pool, has been developed to analyze the fluid flow in the mold region of a continuous slab caster as shown schematically in Fig. 6. Generally, one quarter of the mold needs to be modeled because of assumption of twofold symmetry. However, in the present problem, the whole mold is modeled to investigate asymmetric nature of flow. The flow is highly turbulent, even far away from the nozzle, as indicated by the calculated Reynolds number more than 10000. Lam and Bremhorst $^{8)}$ proposed that the Low-Reynolds-number turbulent model is very convenient for implementation, since the wall function is not needed near mold wall. The elliptic time-averaged transport equations governing this three-dimensional flow are of the following general form:

$$
\nabla \cdot(\rho \mathbf{V} \phi)=\nabla \cdot\left(\Gamma_{\phi} \nabla \phi\right)+S_{\phi}
$$

where $\mathbf{V}$ is the velocity vector, with its three components $u, v$ and $w$ representing the time-averaged velocities in the $x, y$ and $z$ directions, respectively. The variable $\phi$ represents various time-averaged quantities, i.e., mean velocities, turbulence kinetic energy $(k)$, and turbulence energy dissipation rate $(\varepsilon) . \Gamma_{\phi}$ represents the diffusivity of the transported variable $\phi$. Pressure force, body force, generation, and destruction are contained in the source term, $S_{\phi}$. The various transport equations are summarized in Table 2.

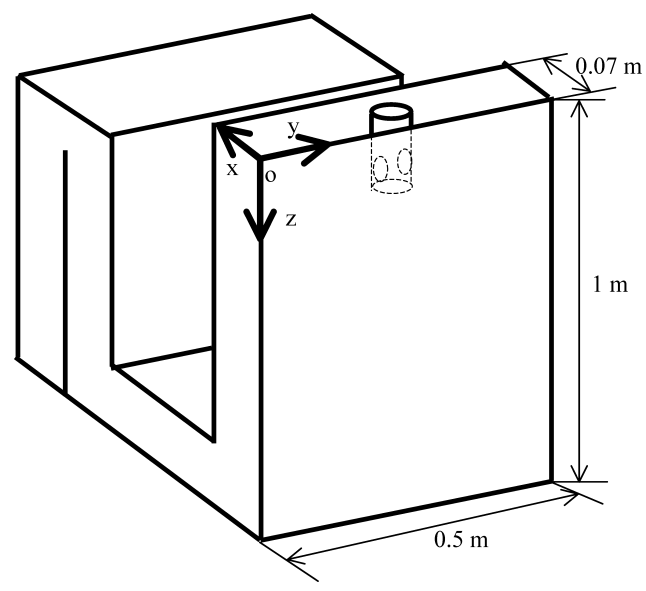

Fig. 6. The snake-shaped mold used in experiment.

In order to describe the behavior of vortices in flow field, definitions of the vorticity $\omega_{z}$ is expressed as follows, respectively.

$$
\omega_{z}=\frac{1}{2}\left(\frac{\partial u_{y}}{\partial x}-\frac{\partial u_{x}}{\partial \theta}\right) \quad \text { in Cartesian coordinates }
$$

or

$$
\omega_{z}=\frac{1}{2}\left\{\frac{1}{r}\left[\frac{\partial}{\partial r}\left(r u_{\theta}\right)-\frac{\partial u_{r}}{\partial \theta}\right]\right\}
$$

in Cylindrical coordinates ...

if the last term in Eq. (3) is neglected, a visual determination is that the intensity of a vortex depends on angular velocity gradient in radial direction and size of a vortex is the area of vortexing flow.

\subsection{Boundary Conditions}

Nozzle inlet velocity $V_{\text {in }}$ is calculated by transferring casting speed $V_{\mathrm{C}}$ on the continuity of flow rate of fluid. At the SEN outlet, the effect of a slide gate or stopper rod on formation of biased flow is excluded. The turbulent kinetic energy and the rate of turbulent kinetic energy dissipation at the inlet are estimated by using the semi-empirical relations, ${ }^{9-10)} k=0.01 V_{\text {in }}^{2}$ and $\varepsilon=2 k^{1.5} / d_{\text {nozzle }}$, where $d_{\text {nozzle }}$ is the nozzle hydraulic diameter. At the free surface, the normal gradients of all variables are set to zero, except that the velocity perpendicular to the surface is zero. The vortex of numerical model is weaker than observed one, because of the assumption of a fixed free surface. As the result of not allowing the free surface to deform, the downward flow velocity can be reduced. This is because the height of the surface waves, which is a part of driving force of the downward flow velocity in vortex, is neglected. At the exit of the mold, fully developed flow is assumed, i.e. normal gradients of all variables are set to zero.

\subsection{Solution Method}

Discretization of equations is conducted by using the control volume integral method. The non-linear coupled equations are solved with the SIMPLEC algorithm. The nozzle volume is taken into account by using the blockage technique. ${ }^{9)}$ The cylindrical nozzle is assumed to be rectan- 
Table 2. Variables in the common equation.

\begin{tabular}{|c|c|c|c|}
\hline Equations & $\phi$ & $\Gamma$ & $S_{\phi}$ \\
\hline Continuity & 1 & 0 & 0 \\
\hline $\mathrm{x}$-direction momentum & $U$ & $\mu_{\mathrm{e}}$ & $S_{u}=\frac{\partial}{\partial x}\left(\mu_{e} \frac{\partial u}{\partial x}\right)+\frac{\partial}{\partial y}\left(\mu_{e} \frac{\partial v}{\partial x}\right)+\frac{\partial}{\partial z}\left(\mu_{e} \frac{\partial w}{\partial x}\right)-\frac{\partial p}{\partial x}$ \\
\hline $\mathrm{y}$-direction momentum & V & $\mu_{\mathrm{e}}$ & $S_{v}=\frac{\partial}{\partial x}\left(\mu_{e} \frac{\partial u}{\partial y}\right)+\frac{\partial}{\partial y}\left(\mu_{e} \frac{\partial v}{\partial y}\right)+\frac{\partial}{\partial z}\left(\mu_{e} \frac{\partial w}{\partial y}\right)-\frac{\partial p}{\partial y}$ \\
\hline z-direction momentum & $W$ & $\mu_{\mathrm{e}}$ & $S_{w}=\frac{\partial}{\partial x}\left(\mu_{e} \frac{\partial u}{\partial z}\right)+\frac{\partial}{\partial y}\left(\mu_{e} \frac{\partial v}{\partial z}\right)+\frac{\partial}{\partial z}\left(\mu_{e} \frac{\partial v}{\partial z}\right)-\frac{\partial p}{\partial z}+\rho \mathrm{g}$ \\
\hline $\begin{array}{l}\text { Turbulent kinetic } \\
\text { Energy }\end{array}$ & K & $\frac{\mu_{t}}{\sigma_{k}}$ & $\begin{aligned} S_{k}= & G-\rho \varepsilon \\
G= & \mu_{t}\left\{2\left(\frac{\partial u}{\partial x}\right)^{2}+2\left(\frac{\partial v}{\partial y}\right)^{2}+2\left(\frac{\partial w}{\partial z}\right)^{2}\right. \\
& \left.+\left(\frac{\partial u}{\partial y}+\frac{\partial v}{\partial x}\right)^{2}+\left(\frac{\partial u}{\partial z}+\frac{\partial w}{\partial x}\right)^{2}+\left(\frac{\partial w}{\partial y}+\frac{\partial v}{\partial z}\right)^{2}\right\}\end{aligned}$ \\
\hline $\begin{array}{l}\text { Dissipation rate of } \\
\text { turbulent kinetic energy }\end{array}$ & $\varepsilon$ & $\frac{\mu_{t}}{\sigma_{\varepsilon}}$ & $S_{\varepsilon}=C_{1} f_{1} G \frac{\varepsilon}{k}-C_{2} f_{2} \rho \frac{\varepsilon^{2}}{k}$ \\
\hline \multicolumn{4}{|c|}{ Effective viscosity ${ }^{8)}: \mu_{t}=\frac{C_{\mu} f_{\mu} \rho k^{2}}{\varepsilon}, \mu_{\mathrm{e}}=\mu+\mu_{t}$, } \\
\hline & & & ${ }_{\mu}=0.09, \sigma_{\mathrm{k}}=1.0, \sigma_{\varepsilon}=1.3, \sigma_{\mathrm{C}}=1.0$ \\
\hline
\end{tabular}

gle so as to apply conveniently the blockage technique. A combination of an Alternating-Direction-semi-Implicit iteration scheme (ADI) and block correction is used to resolve the discretized nonlinear differentiate equations into algebraic equations. A FORTRAN computer code was independently developed for this problem. The $62 \times 24 \times 82$ mesh system is used as main mesh system after grid refinement of solutions. ${ }^{10)}$ When the maximum relative error between successive solutions falls below $0.1 \%$, the iterations are stopped and reasonably converged velocity and turbulence fields for this problem can be obtained. Over 3000 iterations are required to reach this starting from an initial guess of zero velocity.

\section{Results and Discussion}

Numerical studies are designed to develop a better understanding of the vortexing flow phenomena in the mold. The present simulation is based the idea that the existence of vortices is the result of three-dimensional biased flow in the mold. Figure 7 shows the computed velocity distribution of a half thickness and free surface while the SEN is located on the mold centerline. It can be observed that the flow field has twofold symmetry, and there are no vortices on the free surface. However, in practical case, the existence of an absolutely twofold symmetric flow field is almost impossible to achieve, any small perturbation causes the occurrence of biased flow.

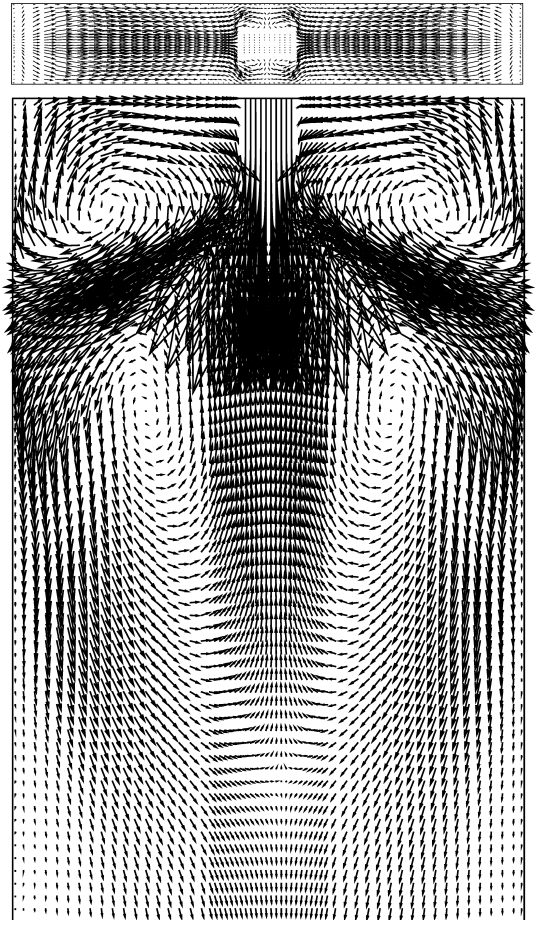

Fig. 7. Calculated flow field in the free surface and half thickness of slab in case that the nozzle is located at the center of mold, maximum velocity is at inlet of nozzle, $V_{\text {in }}=2 \mathrm{~m} / \mathrm{s}$ and casting speed is $V_{\mathrm{C}}=3 \mathrm{~m} / \mathrm{min}$.

4.1. The Generation of the Vortices and Compare with the Experiment

In numerical simulation, the vortexing flow is reproduced 


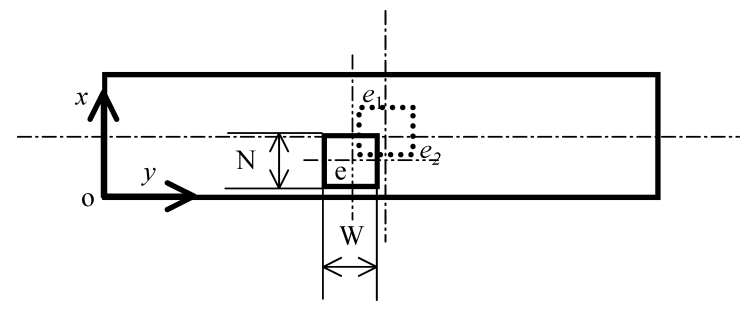

Fig. 8. The location of nozzle used in simulation.

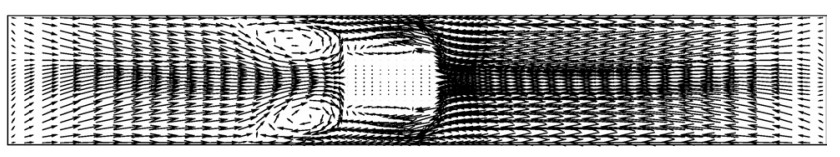

(a)

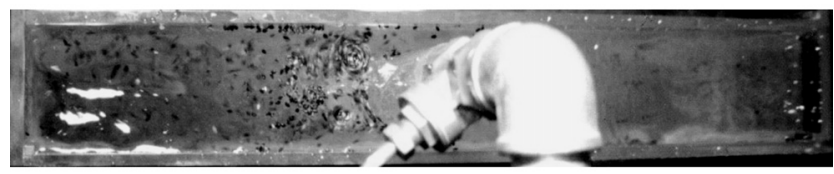

(b)

Fig. 9. Comparison of the two vortices in the surface between (a) simulated and (b) observed, $V_{\mathrm{C}}=3 \mathrm{~m} / \mathrm{min}, e_{1}=15 \mathrm{~mm}$ and $e_{2}=0$.

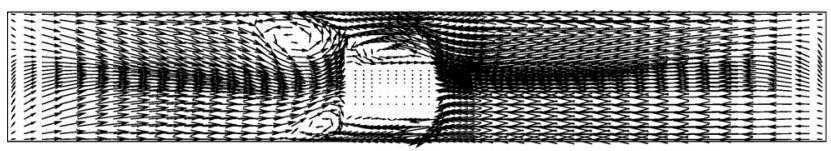

(a)

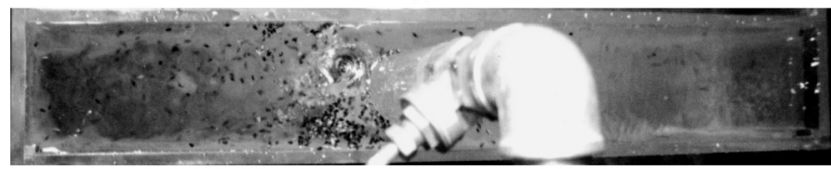

(b)

Fig. 10. Comparison of the vortices in the surface between (a) simulated and (b) observed. $V_{\mathrm{C}}=3 \mathrm{~m} / \mathrm{min}, e_{1}=15 \mathrm{~mm}$ and $e_{2}=5 \mathrm{~mm}$.

by changing the SEN position, i.e. change the SEN biased distances of $e_{1}$ and $e_{2}$ from mold centerline in width and thickness direction, respectively, as shown in Figs. 8 and 9 shows the comparison of two vortices between numerical results and observed one, it is observed that there is a reasonable agreement, although there are differences in size and location of vortices. In fact, such flow patterns have been observed in experiment whether the SEN in center or off- center of the mold, these illustrate that not only the offcenter placing of the SEN produces the vortexing flow but also other factors do it. Nevertheless, only the off-center placing of the SEN is used to produce the vortexing flow in the simulation. An attempt of producing a vortex using biased distances in both of width and thickness direction and comparing with experimental observation is also shown in Fig. 10. A large vortex and a small vortex in free surface of mold are produced in simulation. However, only one vortex is observed in water model experiment. The small vortices result from the assumptions of model.

Figure 11 shows the three-dimensional flow in selected sections of a continuous casting of slab with a $3 \mathrm{~m} / \mathrm{min}$ casting speed. A $15 \mathrm{~mm}$-biased distance in width direction and a $5 \mathrm{~mm}$ biased distance in thickness direction of the

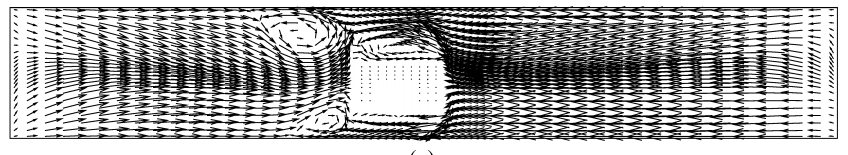

(a)

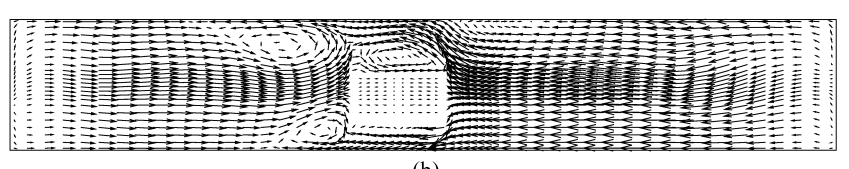

(b)

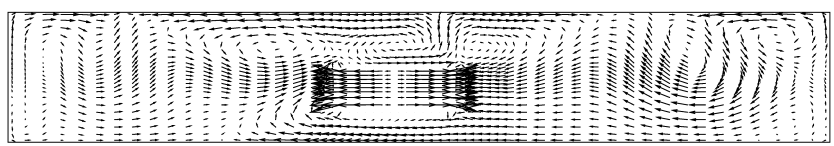

(c)

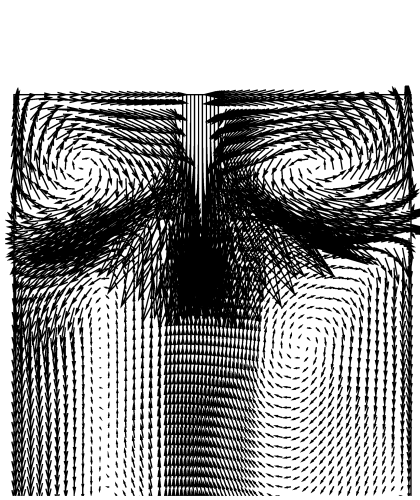

(d)

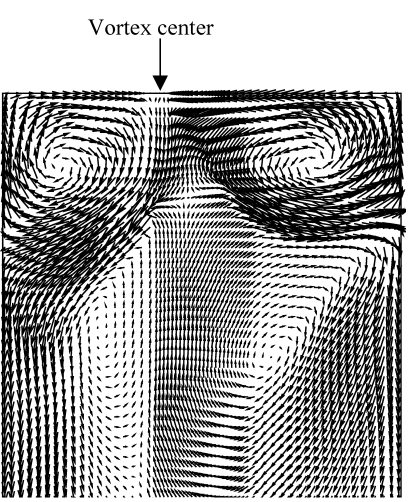

(e)
Fig. 11. Calculated flow field in mold, $V_{\mathrm{C}}=3 \mathrm{~m} / \mathrm{min}, e_{1}=15 \mathrm{~mm}$ and $e_{2}=5 \mathrm{~mm}$. The selected sections (a) $z=0$, (b) $z=50 \mathrm{~mm}$, (c) $z=100 \mathrm{~mm}$ and (d) half thickness of the mold, (e) The section paralleling wide face and through the center of vortex, $x=53 \mathrm{~mm}$.

SEN from the mold centerline, which are $3 \%$ of mold width and $7 \%$ of mold thickness, are assumed to produce the vortexing flow. The nozzle is always displaced to the left of mold centerline in the present work. Figs. 11(a), 11(b) and 11(c) show the flow patterns on three horizontal sections below the free surface and a vortex adjacent to the SEN is clearly observed. The flow velocities of the two sides around the SEN are different. A large vortex is located at the low velocity side adjacent to the SEN. The flow from the high velocity side through the back of the SEN meets the flow from the low velocity side, and shearing takes place to form a vortex. The vortex depth reach the level of the SEN outlet where the vortexing flow is broken by the jet discharged from the SEN. The vortex strength depends on the local horizontal velocity of molten steel, and decreases gradually with distance from the free surface. The flow field of the half thickness of slab is shown in Fig. 11(d), where an asymmetrical flow pattern can be clearly observed. First, sizes of recirculation zones on two sides of the SEN are not the same. On the left side, i.e. the narrowing side, the lower recirculation zone is expanded in the depth direction; and on the right side, i.e. the widening side, the lower recirculation zone is expanded in the width direction. Second, the lower recirculating flow of the left side and the upper recirculating flow of the right side become stronger than the others. The flow field in the vertical section going though the centerline of the large vortex and paralleling the wide face is shown in Fig. 11(e). A downward 
flow is observed in the vortex region.

\subsection{Effects of Off-centerness of the SEN and Casting Speed}

Figure 12 illustrates the effects of off-centerness of the SEN and increasing of casting speed on vortexing flow in the mold. With the SEN deviation from the centerline of the mold, the vortexing zone is gradually expanded because a stronger biased flow occurs. The size of vortices varies also with the moving of SEN toward wide face wall of the mold. The observed sizes of vortices are not obvious with the location of the SEN such as simulation, but the intensity of vortices increases. In fact, some factors such as the unbalance outflow rate from the SEN, transient flow fluctuation and stochastic turbulence etc. affect the vortexing flow, but only the off-center placing of the SEN is considered. Monotonous change may be seen in Fig. 12. A trend toward the reinforcement of vortexing flow with an increase of casting speed can be observed. The reason is the increase of the surface velocity and downward velocity as shown in Fig. 12. Nevertheless, the size of the vortexing zone and flow structure are the same as that of the other casting speed. According to the simulations, it can be summarized that size of the vortices depends on the biased distance of the SEN, and the intensity of vortexing flow depends on the biased distance of the SEN and casting speed of the continuous caster.

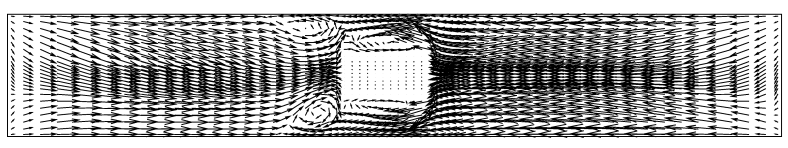

(a)

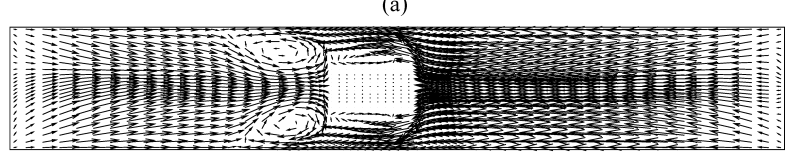

(b)

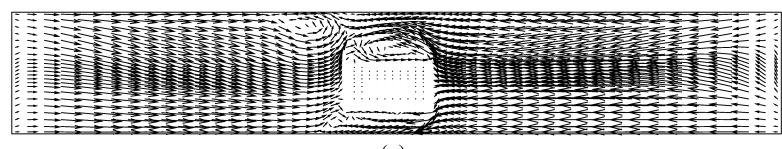

(c)

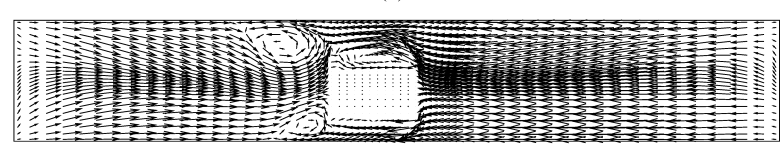

(d)

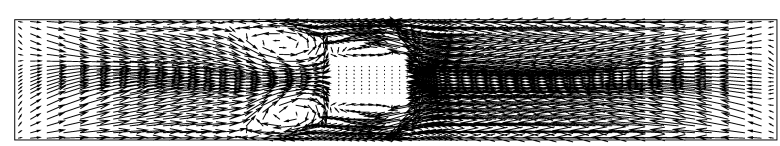

(e)

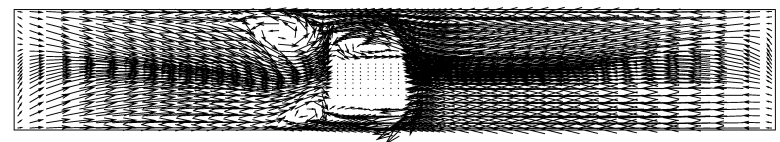

(f)

Fig. 12. The effects of the location of the SEN and casting speed on flow field in the free surface, the maximum velocity of (a) though (f) is $0.61 \mathrm{~m} / \mathrm{s}$. (a) $V_{\mathrm{C}}=3 \mathrm{~m} / \mathrm{min}, e_{1}=5 \mathrm{~mm}$ and $e_{2}=0$; (b) $V_{\mathrm{C}}=3 \mathrm{~m} / \mathrm{min}, e_{1}=15 \mathrm{~mm}$ and $e_{2}=0$; (c) $V_{\mathrm{C}}=3 \mathrm{~m} / \mathrm{min}, e_{1}=5 \mathrm{~mm}$ and $e_{2}=5 \mathrm{~mm}$; (d) $V_{\mathrm{C}}=3 \mathrm{~m} / \mathrm{min}$, $e_{1}=15 \mathrm{~mm}$ and $e_{2}=5 \mathrm{~mm}$; (e) $V_{\mathrm{C}}=4 \mathrm{~m} / \mathrm{min}, e_{1}=15 \mathrm{~mm}$ and $e_{2}=0$; (f) $V_{\mathrm{C}}=4 \mathrm{~m} / \mathrm{min}, e_{1}=15 \mathrm{~mm}$ and $e_{2}=5 \mathrm{~mm}$.

\subsection{Vortex Suppressing Ways}

From the foregoing discussion, the mechanism of vortex formation on the surface of molten steel in the mold may be summarized as follows. Vortexing is the result of shearing of the two surface flows from the mold narrow faces when they meet adjacent to the SEN. Vortexing flow is composed of rotation of fluid in the horizontal sections and downward flow. The vortex intensity increases with increasing surface velocity. On the basis of these mechanism, the suppression methods of vortexing are suggested in two ways: one is to suppress the surface velocity and the other is to remove the downward flow near the SEN. Magnetic field application and argon gas injection are employed to achieve these objectives.

\section{Conclusions}

(1) Changes the shape of a vortex or two vortices with time were observed and recorded in photographs in water model experiment with a snake-shaped mold. Although the geometrical and operating conditions are symmetry, the vortex formation under the conditions of the transient and high casting speed cannot be avoided. These indicate that not only the off-center placing of the SEN produce the vortexing flow but also other factors do it. With the outflow angle of the SEN change from downward to upward, the trend toward the vortices formation is stronger and stronger.

(2) A mathematical model has been developed to analyze vortexing flow in the slab continuous casting mold. Moving the SEN from center to off-center reproduced the vortexing flow. The reasonable agreement of vortices between numerical results and observation was obtained.

(3) Two vortices occur when the SEN is located at the off-center of the mold only in the width direction. If the SEN is located at the off-center of the mold in both the width direction and thickness direction, a large vortex and two small vortices were produced, the large vortex accords with the experimental observation.

(4) The size of the vortexing zone depended on the offcenterness of the SEN, and the intensity of vortexing flow depended on both of the off-centerness of the SEN and casting speed of the continuous caster.

\section{Nomenclature}

$e: \quad$ Biased distance of nozzle $(\mathrm{mm})$

g: Acceleration due to gravity $\left(\mathrm{m} / \mathrm{s}^{2}\right)$

$k$ : Turbulent kinetic energy $\left(\mathrm{m}^{2} / \mathrm{s}^{2}\right)$

$L_{\mathrm{n}}: \quad$ Nozzle submergence depth $(\mathrm{mm})$

$N$ : Thickness of nozzle $(\mathrm{mm})$

$p$ : Pressure $\left(\mathrm{N} / \mathrm{m}^{2}\right)$

$T_{0}$ : $\quad$ Casting temperature $(\mathrm{K})$

$R_{\mathrm{t}}$ : Turbulent Reynolds number, defined as $R_{\mathrm{t}}=\rho k^{2} / \mu \varepsilon$

$R_{\mathrm{w}}$ : Turbulent Reynolds number, defined as $R_{w}=$ $\rho k^{1 / 2} y_{\mathrm{w}} / \mu$

$u, v, w$ : Velocity components $(\mathrm{m} / \mathrm{s})$

$W$ : Width of nozzle $(\mathrm{mm})$

$V_{\mathrm{C}}$ : The casting speed $(\mathrm{m} / \mathrm{s})$

$V_{\text {in }}$ : The inlet velocity of nozzle $(\mathrm{m} / \mathrm{s})$

$y_{\mathrm{w}}:$ The distance from wall $(\mathrm{m})$ 
$x, y, z: \quad$ The Cartesian coordinates (m)

$\alpha$ : Gas volume fraction

$\varepsilon$ : Rate of turbulent kinetic energy dissipation $\left(\mathrm{m}^{2} / \mathrm{s}^{2}\right)$

$\mu_{1}, \mu_{\mathrm{t}}, \mu_{\mathrm{e}}$ : Laminar, turbulent, and effective viscosities $\left(\mathrm{N} \cdot \mathrm{s} / \mathrm{m}^{2}\right)$

$\rho, \rho_{\mathrm{g}}, \rho_{\mathrm{liq}}:$ Mixture, gas, and liquid densities $\left(\mathrm{kg} / \mathrm{m}^{3}\right)$

$\sigma_{\partial}$ : Turbulent Schmidt number

$\sigma_{\mathrm{g} 0}$ : Gas volume fraction at inlet

$\sigma_{k}, \sigma_{\varepsilon}:$ Schmidt number for turbulent kinetic energy and its dissipation rate

\section{Acknowledgment}

One of the authors, Baokuan Li, is grateful to the National Natural Science Foundation of China for support of this reseach, project No. 50274029.

\section{REFERENCES}

1) Y. H. Wang: 73rd Steelmaking Conf. Proc., ISS-AIME, Warrendale, PA, (1990), 473 .

2) Q. L. He: ISIJ Int., 33 (1993), 343.

3) M. Gebhard, Q. L. He and J. Herbertson: 76th Steelmaking Conf. Proc., ISS-AIME, Warrendale, PA, (1993), 441.

4) T. Honeyands and J. Herbertson: Steel Res., 66 (1995), 287.

5) E. Gupta and A. K. Lahri: Metall. Trans. B, 27B (1996), 757.

6) X. Huang and B. G. Thomas: Can. Metall. Q., 37 (1998), 197.

7) J. Yoshida, M. Iguchi and S. Yokoya: Tetsu-to-Hagané, 87 (2001), 529.

8) C. K. G. Lam and K. Bremhorst: J. Fluid Eng., 103 (1981), 456.

9) B. K. Li, T. Okane and T. Umeda: Metall. Trans. B, 31B (2000), 1491.

10) B. K. Li, T. Okane and T. Umeda: Metall. Trans. B, 32B (2001), 1053. 